\title{
Collection and Analysis of Vegetative Cover Data
}

R. H. Sauer

S. L. Owzarski

July 1979

Prepared for the U.S. Department of Energy under Contract EY-76-C-06-1830

Pacific Northwest Laboratory Operated for the U.S. Department of Energy by Battelle Memorial Institute 


\title{
NOTICE
}

This report was prepared as an account of work sponsored by the United States Government. Neither the United States nor the Department of Energy, nor any of their employees, nor any of their contractors, subcontractors, or their employees, makes any warranty, express or implied, or assumes any legal liability or responsibility for the accuracy, completeness or usefulness of any information, apparatus, product or process disclosed, or represents that its use would not infringe privately owned rights.

The views, opinions and conclusions contained in this report are those of the contractor and do not necessarily represent those of the United States Government or the United States Department of Energy.

\author{
PACIFIC NORTHWEST LABORATORY \\ operated by \\ BATTELLE \\ for the \\ UNITED STATES DEPARTMENT OF ENERGY \\ Under Contract EY-76-C-06-1830
}

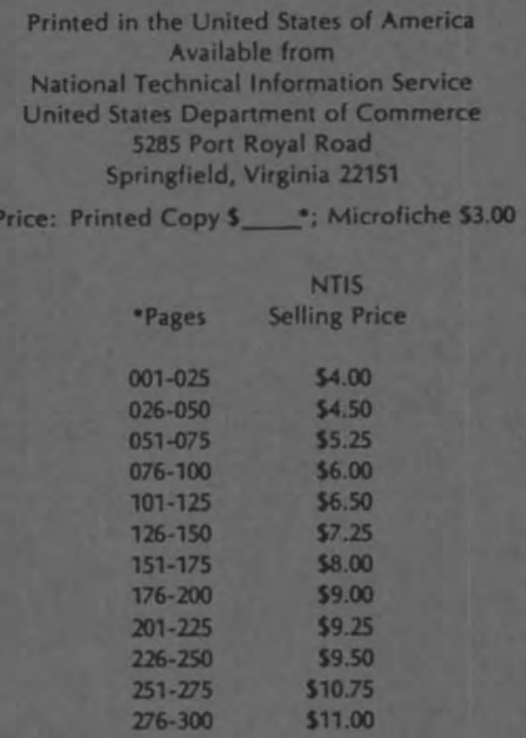


R. H. Sauer

S. L. Owzarski

JuTy 1979

Prepared for

the U.S. Department of Energy

under Contract EY-76-C-06-1830

Pac if ic Northwest Laboratory

Richland, Washington 99352 


\section{CONTENTS}

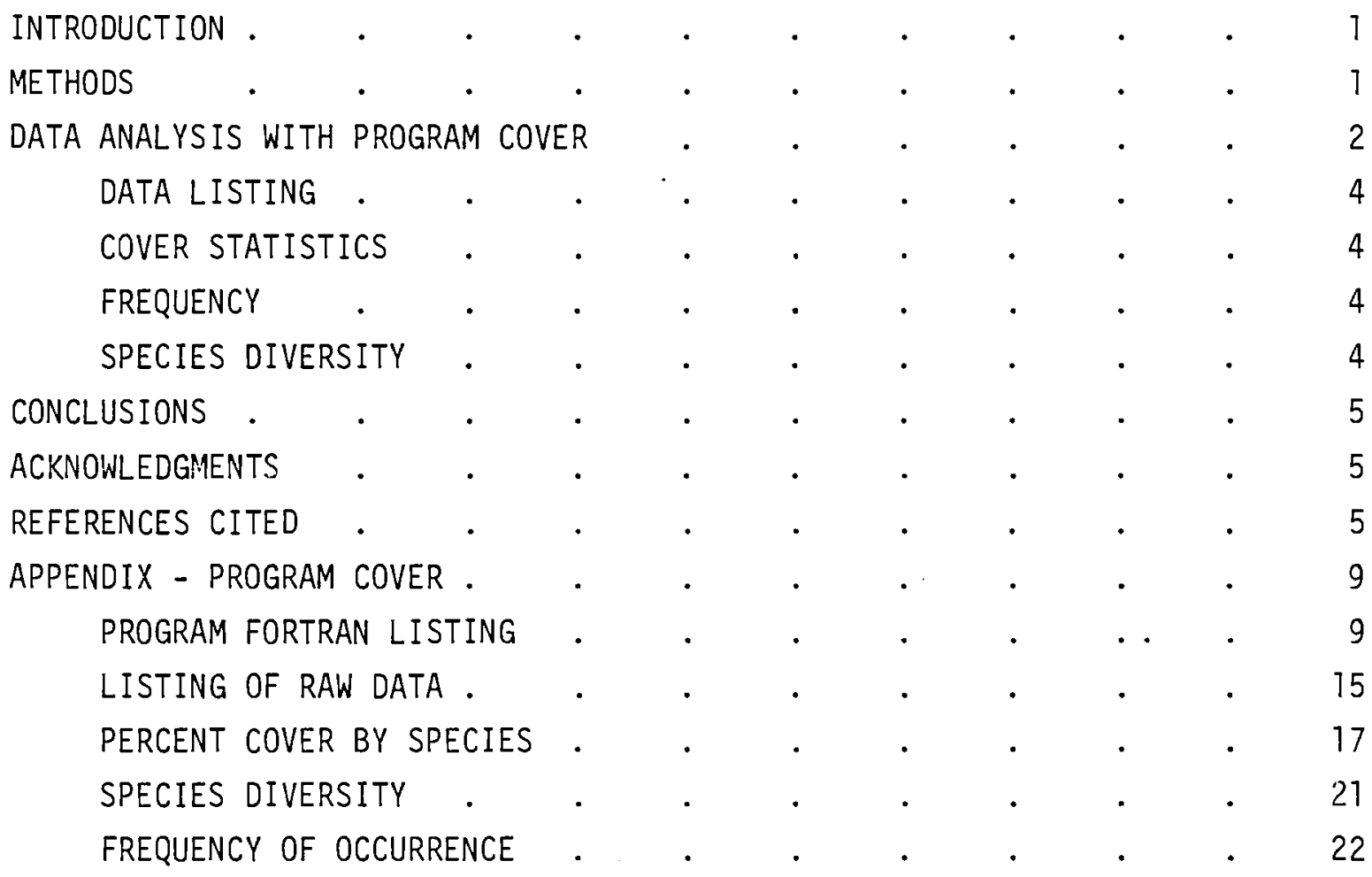




\section{INTRODUCTION}

This program was developed at Pacific Northwest Laboratory to facilitate the anaysis of vegetative data using Daubenmire's (1959) canopy cover approach. It has been used in numerous research programs on the Hanford Site since the program's creation in 1975.

The assemblage of plant species (vegetation) of an area offers unique opportunities to recognize and evaluate changes in the environment. The quantity or persistence of a vegetative cover reflects the growth conditions of the immediate area. If a species cannot adapt to a change in its environment, its abundance will change. This change in abundance can be detected by vegetational analysis and can be used to pinpoint environmental changes. Vegetation then can be thought of as an integrator of the environmental factors that affect plant growth, and as such is a useful indicator of environmental change.

For a specific example let us consider the deposition of salt from a cooling tower. At some time, depending on rate of deposition, enough salt will be deposited on the soil to slow or stop the growth and reproduction of most plant species, and the vegetative cover will become thinner and consist of (probably) fewer and different species, compared to the initial condition. Periodic data collection and analys is will document the change and add to our understanding of the situation which will be useful in subsequent management activities.

The purpose of this document is to present the rationale, methods, and analytic tools that are commonly used to study changes in vegetation on the Hanford Site.

\section{METHODS}

The method of vegetation analys is that has been successful in the Columbia Basin in Washington State is Daubenmire's (1959) canopy cover approach. Canopy cover is the percent of ground surface covered by a vertical projection to the soil of the outer edges of a plant's live parts. Six classes are recognized (Table 1) in use. The percentage cover of each species present is estimated and placed in one of the six classes.

The advantage of placing the cover of a species into broad classes instead of making more accurate estimates is that it allows faster data collection. The error is reduced with many samples (usually 50) because the actual value in some samples will be less than the midpoint (Table 1), and others, greater than the midpoint. The midpoint value is used to calculate the statistics of canopy cover.

The typical procedure is to position a 50 meter tape (line) in the area to be sampled in such a way that it covers a representative portion of the area. Permanent markers at both ends (metal) fence posts are useful if 
TABLE 1. Cover Classes

\begin{tabular}{ccc}
\hline Cover Class & $\%$ Cover & Midpoint \\
\hline 1 & $0-5$ & 2.5 \\
2 & $>5-25$ & 15.0 \\
3 & $>25-50$ & 37.5 \\
4 & $>50-75$ & 62.5 \\
5 & $>75-95$ & 85.0 \\
6 & $>95-100$ & 97.5
\end{tabular}

annual observations are to be made. A $0.1 \mathrm{~m}^{2}$ rectangle $(20 \mathrm{~cm}$ by $50 \mathrm{~cm})$ is placed in the same position at each one-meter mark. In the first plot, at meter mark 1 , the cover class of the canopy cover of each species present is recorded. The process is repeated for all fifty positions along the line. A data form, A-1500-096 (3-78), has been developed for recording COVER data (Figure 1). On this form the date is recorded in Columns 23-30 and the identification of the line (area etc.) is recorded in columns 6-22. The species is recorded in Columns $1-4$ as a 4 letter code. The first two letters are the first two of the genus, and the last two letters are the first two letters of the species (Garrison et a1. 1976). For example, the code for cheatgrass or Bromus tectorum is BRTE. The cover class of the first species

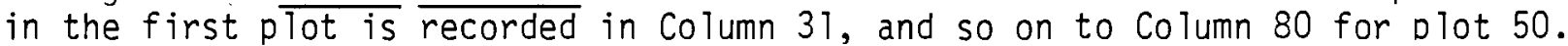
New species are added in Columns 1-4 as encountered. Blanks indicate the species was absent in that plot. A separate data form is used for each 1 ine. The number of lines necessary to sample an area depends on the heterogeneity of the area in question.

\section{DATA ANALYSIS WITH PROGRAM COVER}

The data are ready for analysis after keypunching onto data processing cards. A FORTRAN listing of the program and sample output, consisting of a data listing, frequency, cover statistics, and species diversity for each line is given in the Appendix. In order from the front of the deck are the UNIVAC system control cards, the COVER program described below, and the data arranged in "lines" and "groups." A "line" is the data taken from a single line. A "group" is a collection of "lines" that are to be analyzed together. A group can have between 2 and 10 lines with a maximum of 60 species. Lines within a group are separated by a 9999 card (9's in Columns 1-4). There is no 9999 


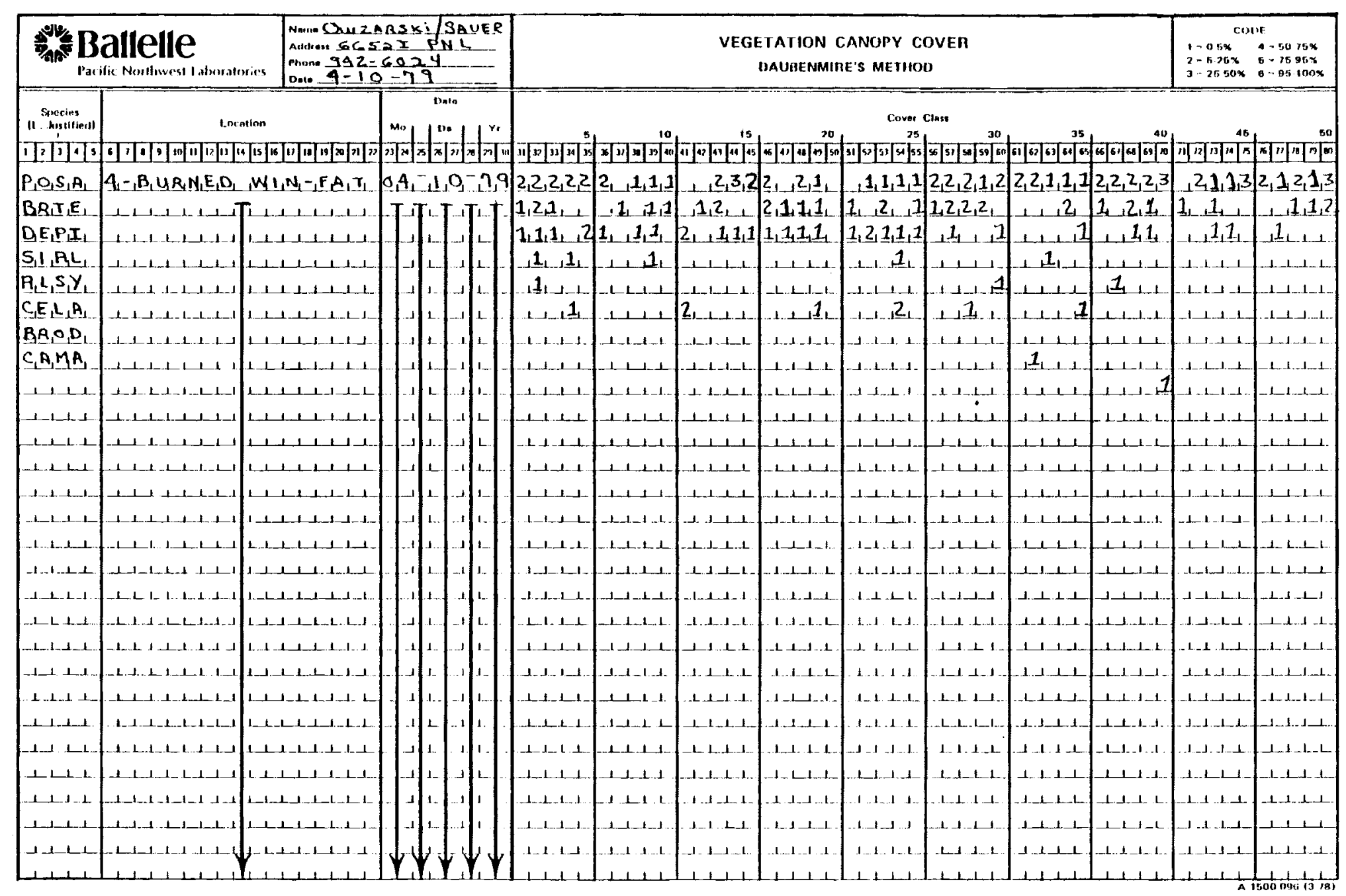

FIGURE 1. Form for Recording Cover Data 
card after the last line. Groups are preceeded by a "number of plots card" on which the number of plots (usually 50) is punched in Columns 2-3. The second card is a "title card" which has 8 columns for a label for each line. For example, Columns 1-8 are for Line 1, etc. Groups are separated by $\$ \$ \$ \$$ cards ( $\$$ in Columns 1-4). There is no $\$ \$ \$ \$$ card after the last group. A word of caution; the program will not operate if there are data cards with species that have no observations on any line. These cards should be removed before running the program.

\section{DATA LISTING}

The data for each group of lines is listed together to facilitate connection of output with input. In the listing, the blanks on the data form indicating that species' absence are printed as zeros.

\section{COVER STATISTICS}

Mean percent cover is listed for each species, identified by the four character code. Beneath each species code the standard deviation (SD), standard error (SE), the coefficient of variation (CV), the upper $95 \%$ confidence limit $(95 \% \mathrm{~V})$ and the lower limit $(95 \% \mathrm{~L})$ are given. Confidence intervals are calculated with the assumption of 50 plots per line. These statistics are for the 50 plots along the line named at the top of the column. The statistics of each line are calculated independently and are presented in a separate column labeled at the top with the name of the 1 ine. The last two columns, labeled "mean" and "SE", are the mean and standard error, respectively, of the various line statistics on the left. Lines are thus combined.

\section{FREQUENCY}

Frequency is a measure of how often a species occurs in the plots along a line. A low frequency indicates that the species is not uniformly distributed. The frequency results are arranged in the same way as the cover statistics.

\section{SPECIES DIVERSITY}

Three indices of species diversity are calculated (Hi11, 1977):

a) Richness or simply the number of species

b) A modified Shannon-Weaver index which is numerically less than the number of species because it is weighted against rare species.

c) A modified Simpson's index which is numerically less than the Shannon-Weaver index because it is further weighted against rare species. 
The numerical values of the species diversity indices are termed the equivalent number of equally common species. These synthetic numbers are useful in theoretical considerations of vegetation structure and function. The modifications and a discussion of species diversity are presented by Hill (1973).

\section{CONCLUSIONS}

We think this method and program COVER are dependable and useful tools in vegetation description and analysis. While we can take no responsibility for program COVER, we ask that any problems and improvements be brought to our attention.

This version of COVER runs on a UNIVAC and was developed on a CYBER 70. We will provide, upon request, a deck of the program to interested parties.

\section{ACKNOWLEDGMENTS}

We gratefully acknowledge the assistance of all members of the Terrestrial Ecology Section and editors of the Ecological Sciences Department at Battelle-Northwest. To Dr. John Rottenberry we express in particular our appreciation for his many helpful suggestions and discussion on species diversity.

\section{REFERENCES CITED}

Daubemire, R. 1959. A Canopy-Coverage Method of Vegetation Analysis. Northwest Science 33:43-64.

Hi11, M.0. 1973. Diversity and Evenness: A Unifying Notation and Its Consequences. Ecology 54:427-432.

Garrison, G. A., J. M. Skovlin, C. E. Poulton and A. H Winward. 1976. Northwest Plant Names and Symbols for Ecosystem Inventory and Analysis. 4th Ed. Pacific Northwest Forest and Range Experiment Station. USDA Forest Service General Technical Report PNW-46. USDA, Forest Service, Port land, Oregon. 
APPENDIX

PROGRAM COVER 
PROGRAM FORTRAN LISTING

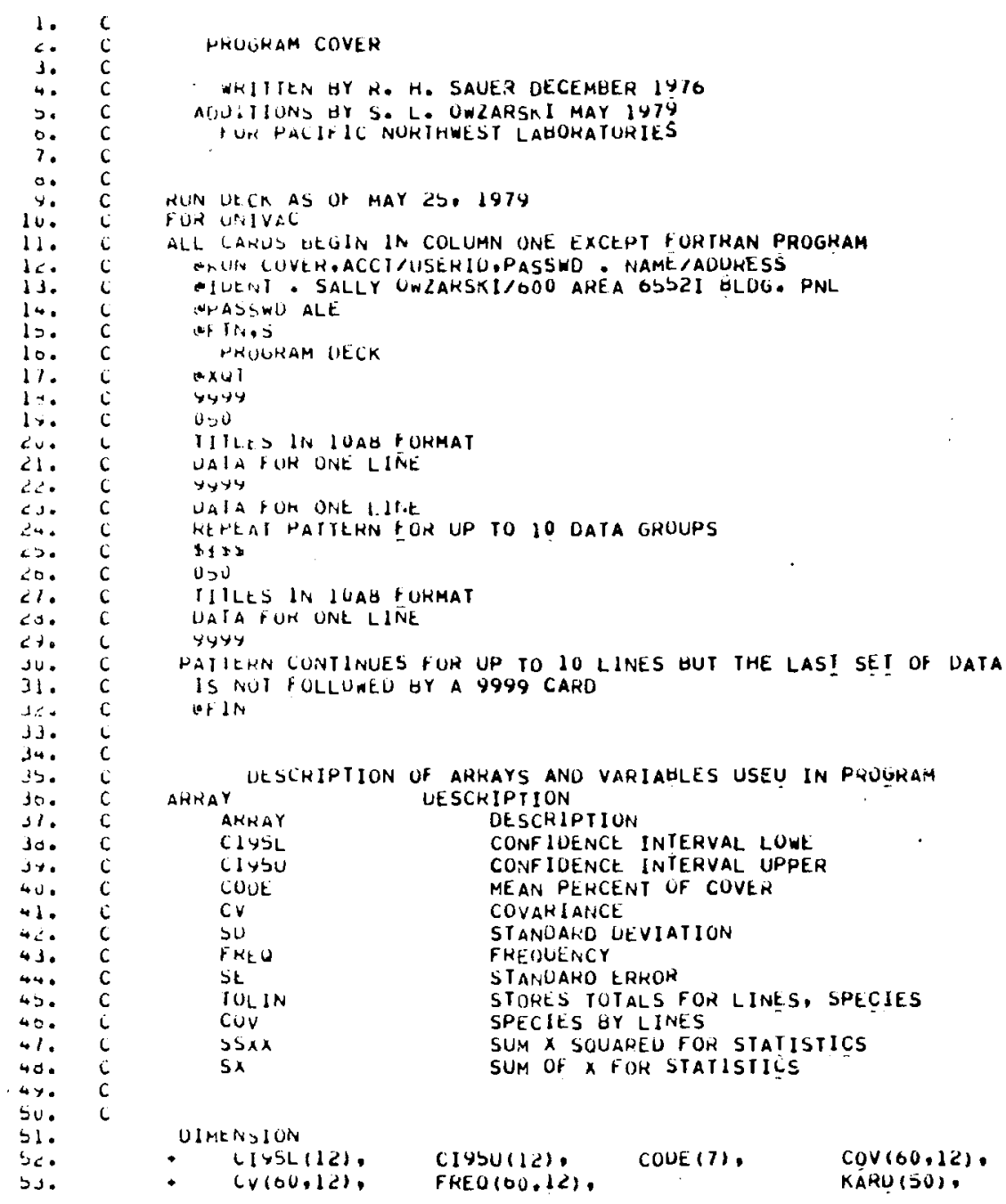




\section{PROGRAM FORTRAN LISTING (Cont inued)}

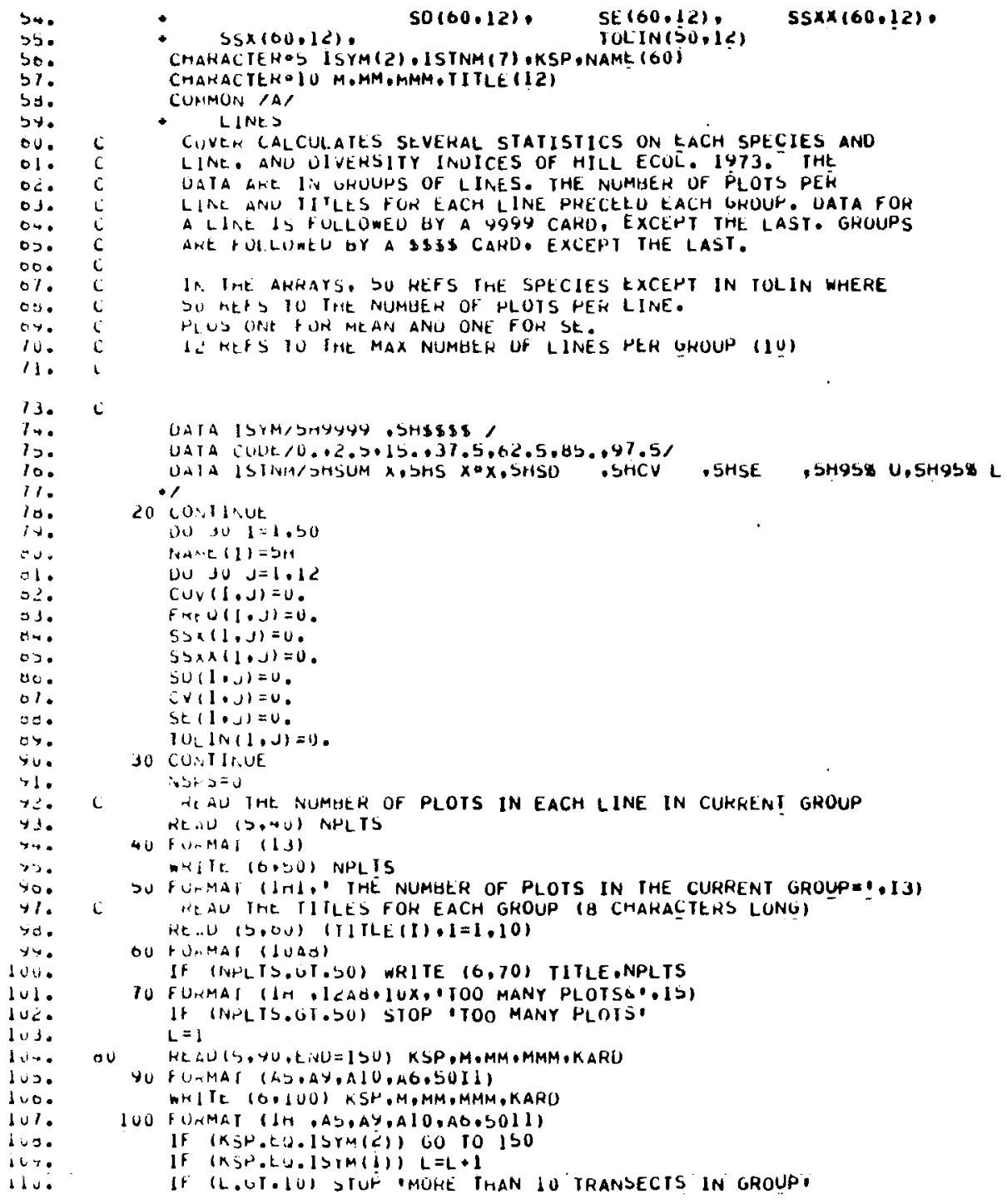


PROGRAM FORTRAN LISTING (Continued)

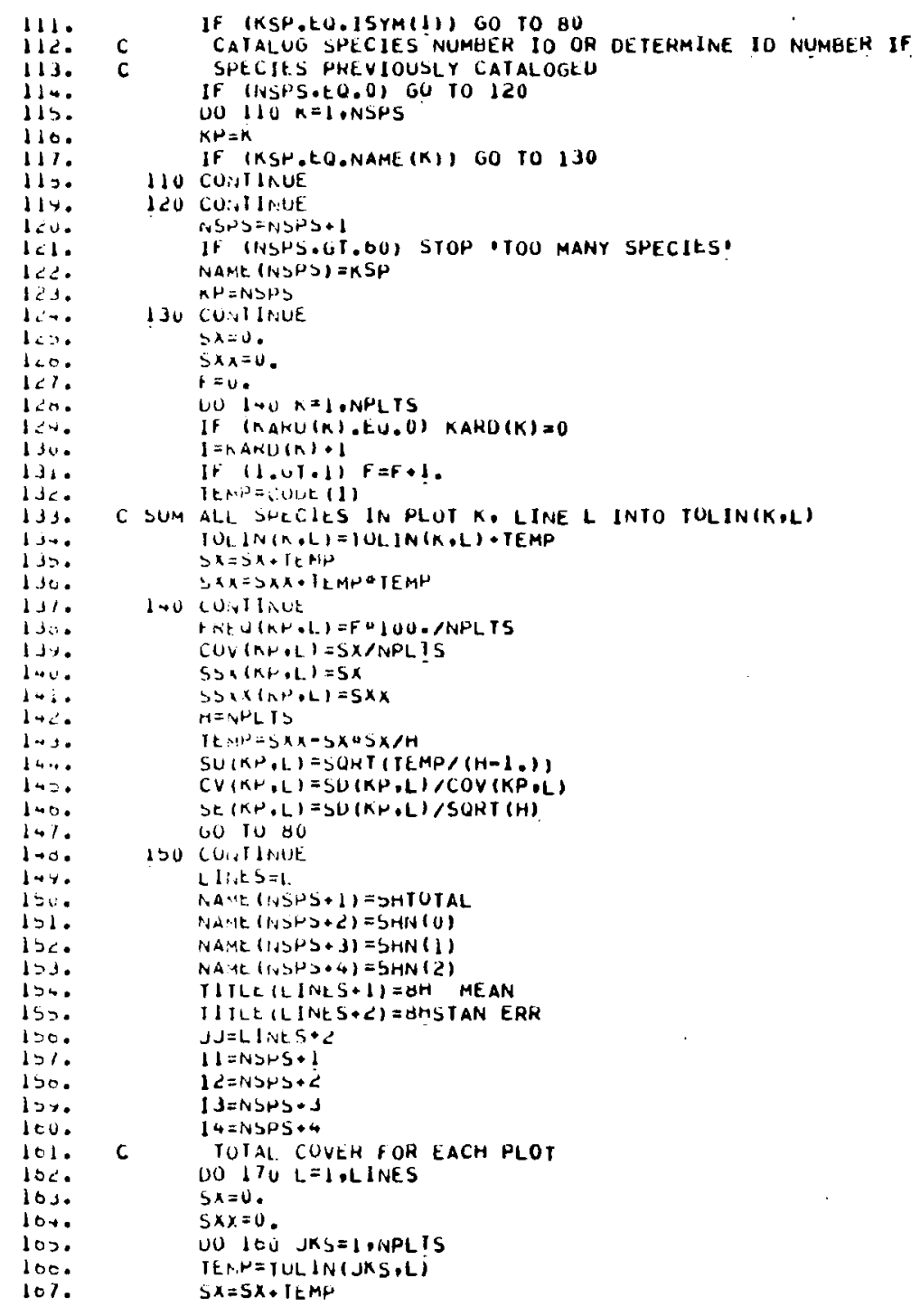




\section{PROGRAM FORTRAN LISTING (Continued)}

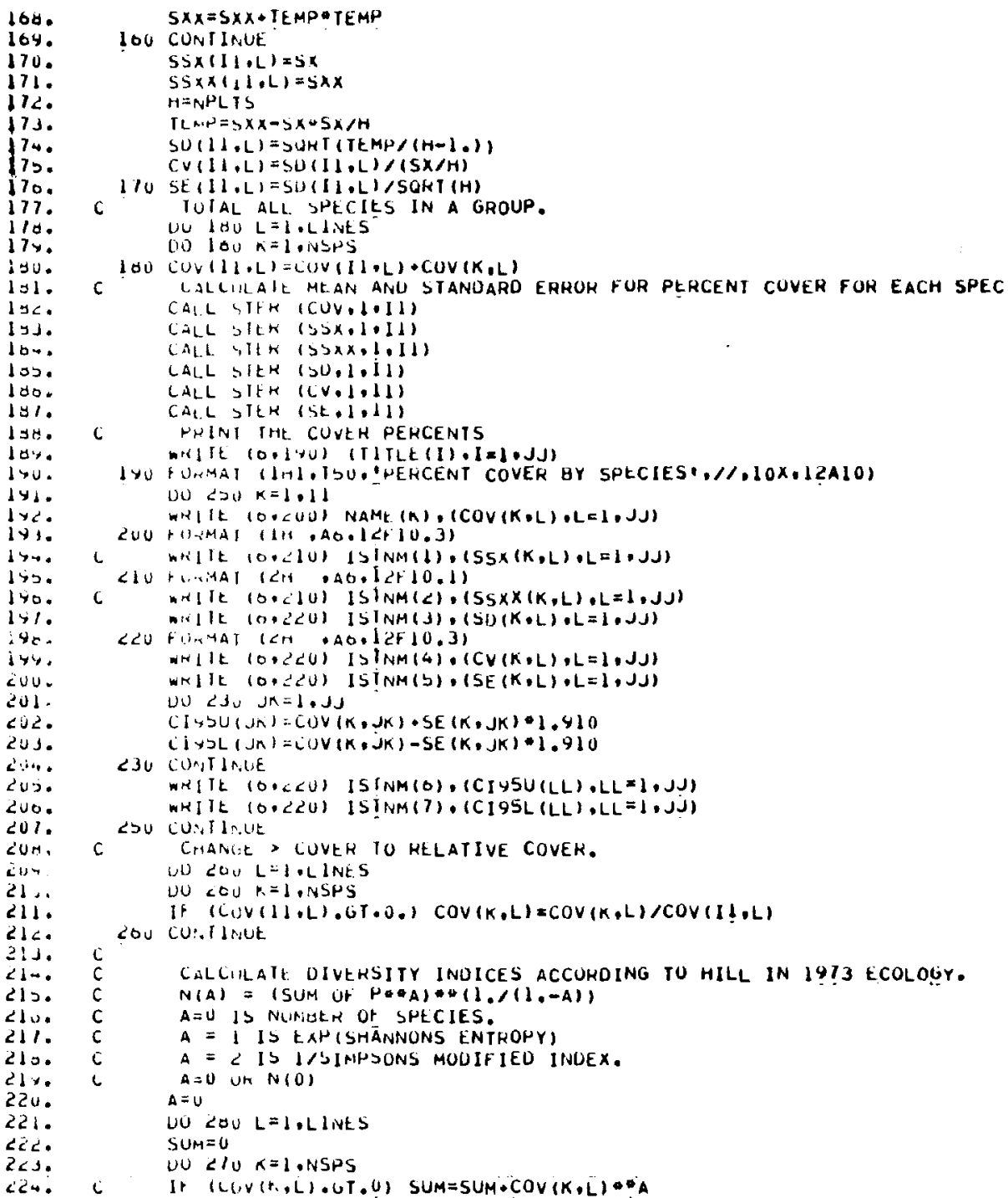




\section{PROGRAM FORTRAN LISTING (Continued)}

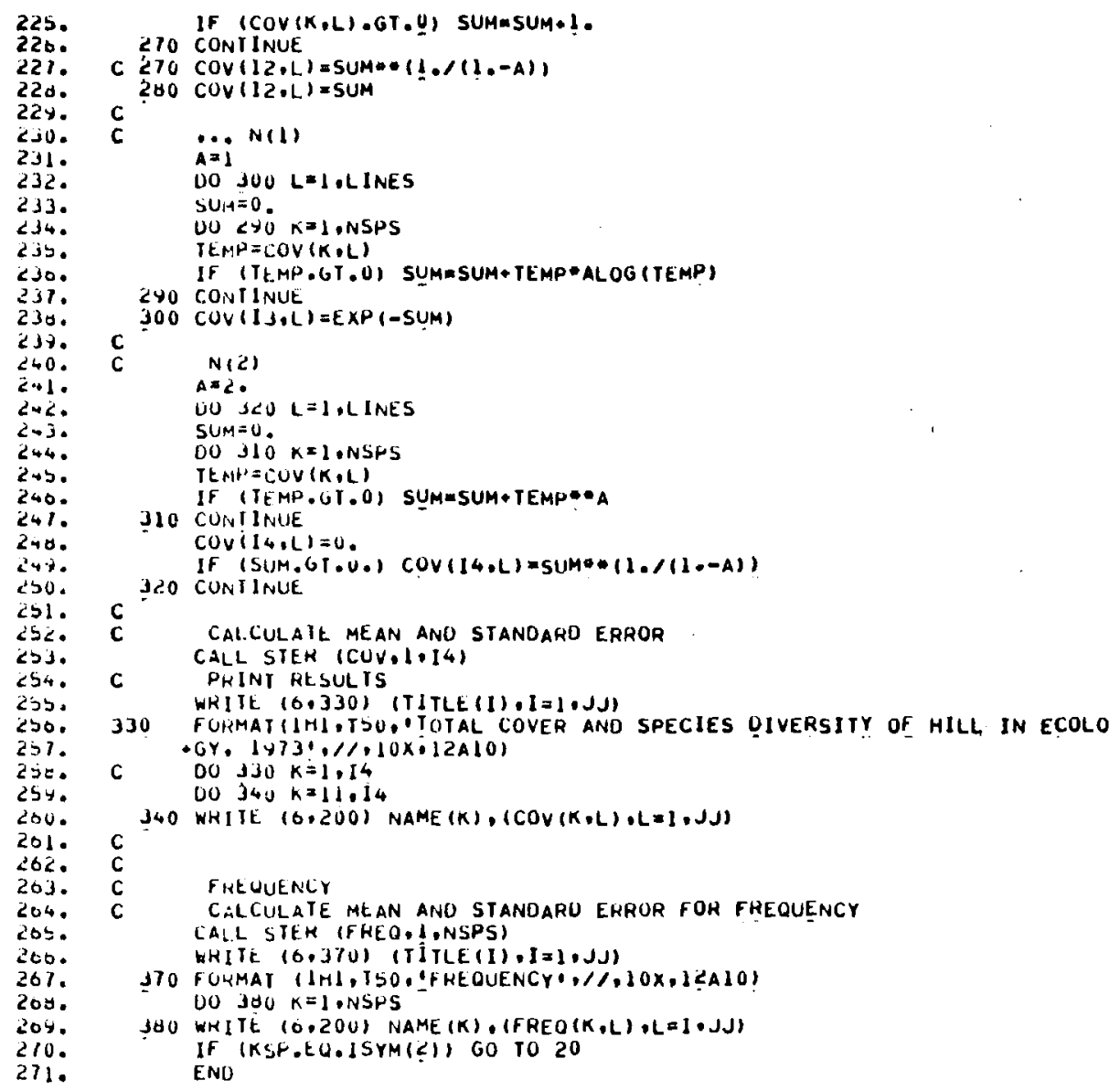


PROGRAM FORTRAN LISTING (Cont inued)

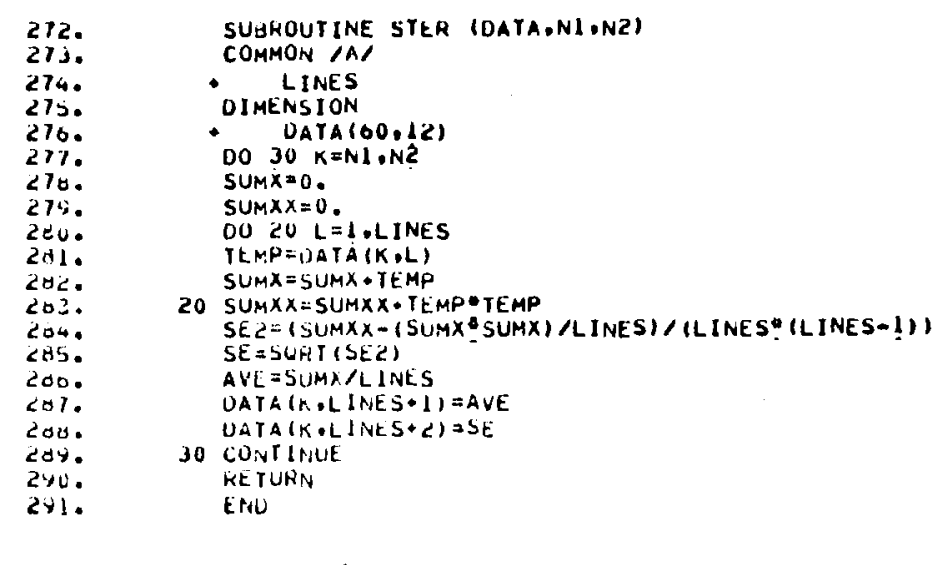




\section{LISTING OF RAW DATA}

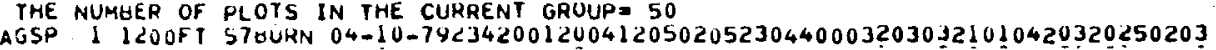
POSA 1 120OFI S7UUKN 04-i0-794J23233233314323323123223234232151123342320024J12022 SHIE 1 12UOF STUUFN 04-10-19201111111101100011101101111011100001100101111000000

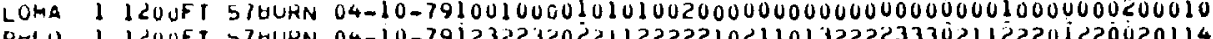

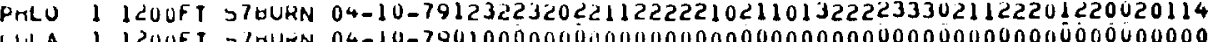
L.ULA I RUUF STOURN O4-10-79O10011

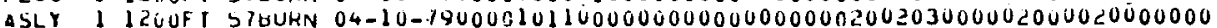
UEFU l LUUF T

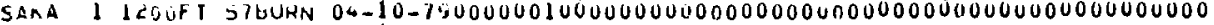
LASE I 120OFT S7DUKN 04-10-7900000001000000010000000010000000000000000060000000

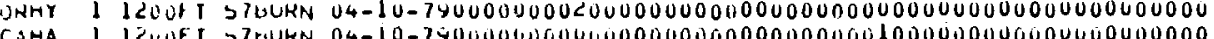

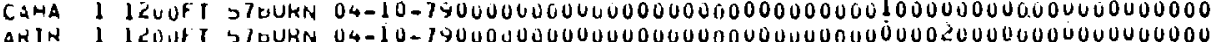
ARIN 1 licUUT SIDURN O4-1 -

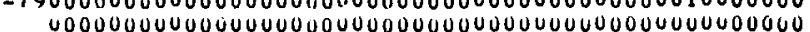

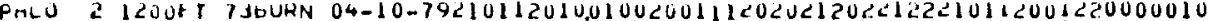

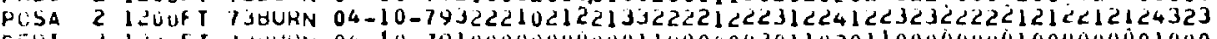
CEPI L LCUUF T ISULIRN 04-10-7910000000000011000600201102011000000001000000001000

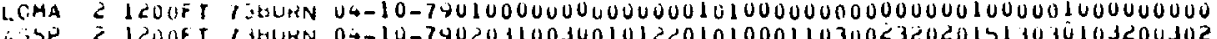

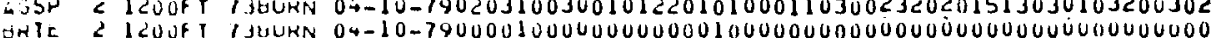
SIAL 2 LCONF T IUUKN 04-10-790000U1006010000000000010010000000000000000000001000

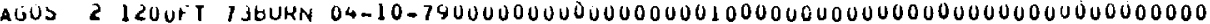

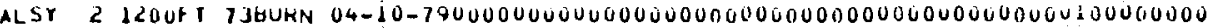
AYYY (1) UNGUAN WNTEATO4-10-790000000030J 200000000000000020000000200000000000100 UNUAN WNT F $2104-10-7932032221322322240233332242333341153222223233302333$

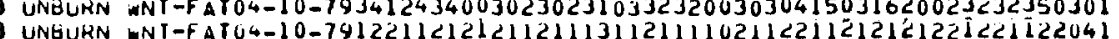

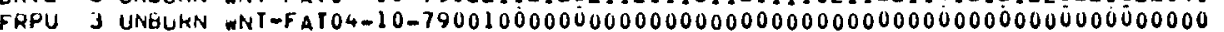
COMA I UNEUFN WNT-FAT04-10-79000110030000000020211000000000000000000201010000000 ALSY 3 UNESUFN WNIFAIO4-10-79000000001000000000000100100003000000000000000000001 SITA 3 UABURN WNTEFAT04-10-7900000000000000230000000000000000000000020000000020000 SAKA 3 UNGUAN WNT-FATO4-1.0-790000000000000100000000000000000000000000000000000000 SIAL 3 UNBUNA WNTFFATO4-I0-790000000000000000010000000000000000000000000000000000 BKOU 3 UNBUAN WNT-FATU4-10-7900000000000000001000000000000000000000000202100000

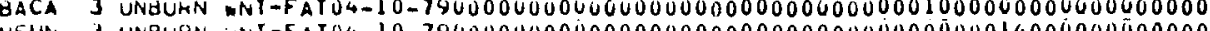

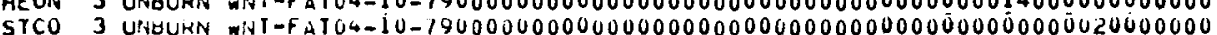

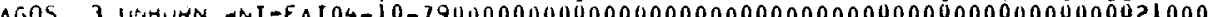
000000000000000000000000000000000000000000000000000

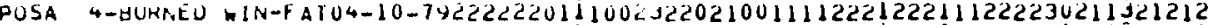
GHIE 4-UURRED MIN-FATO4-10-791210001011012002111010201122<000020102101010000112 DEHI 4-bUKNEU MIN-FATU4-10-79110210110201011110101211001001000001001100011001000 SIAL 4-DUMirE AIN-FAYU-10-79010100001 ILSP 4-BURARE IIIN-FATC4-10-790100000000000000000000000000010000001000000000000 LELA 4-UUKiat.d AIN-FATU4-10-790001000000

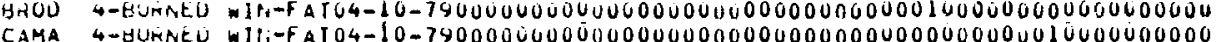
CiYGY

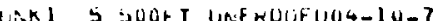

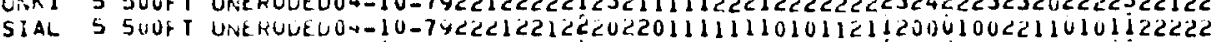

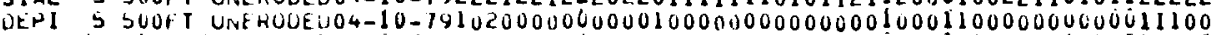

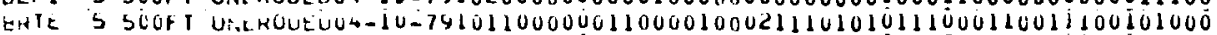




\section{LISTING OF RAW DATA (Continued)}

CHLE 5 SOOFY UNEROUEU04-10-7901101111211111102110001000001000101132101011111221 DAVE S SOUFY UNERODED04-10-79001010000010000000111110011110111111201121111101011

SUOF UNERUUEDO4-10-79000000100000000000100110100000000000200232000000000 SAKA S SUUFI UNERUUED04-10-7900000000001000000000000000001000000000000000000000

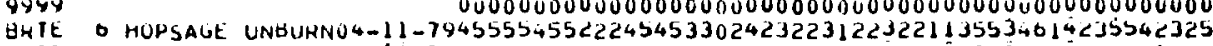
ATSP O RUPAGE UNGUKNO4-11-79<2000 $2000 \$ 0000000050000004100200003000000000001200$ UNKU O TUPSAGE UNBUHNO4-11-79U110100001 U21 11110101002000000000000101120230002 MIGH ORUPSAGE USBUURNUA-11-7900100001001000000100000100110010000100000000004000

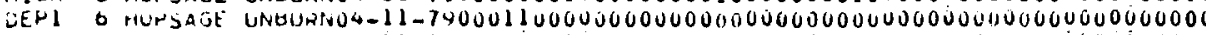

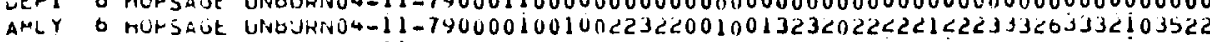

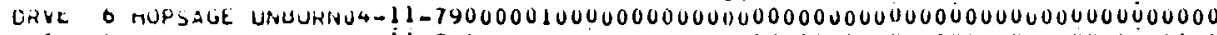

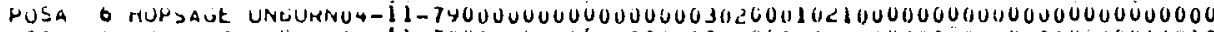
SIAL O HISHSAUE UNGURIN04-11-790000000000000000000000000000000000000000000061011010 GRIE 7 Hit

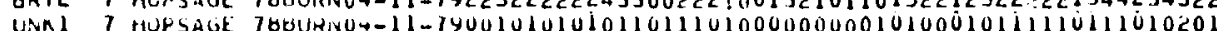

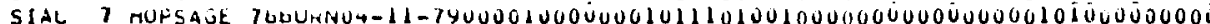

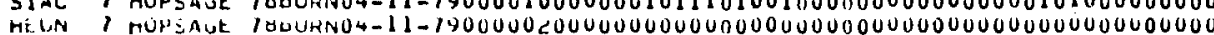

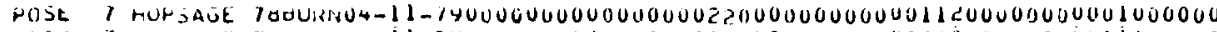

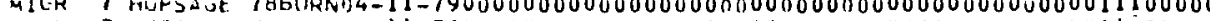
AMLY 7 GUPSAGE TODURNO4-11-79000000000000000000000000000000000000006001000000 g.

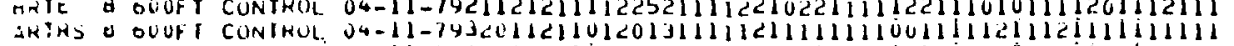

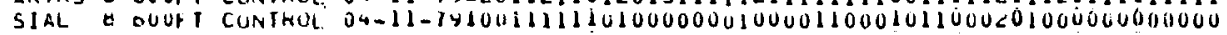

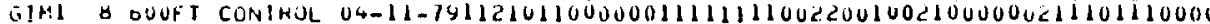
POSA OUOF CUNTHUL O4-11-1900022201020200000002280000200020000010000000000012CO

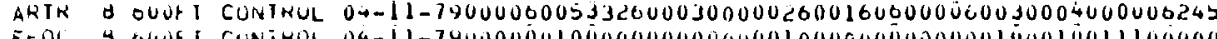

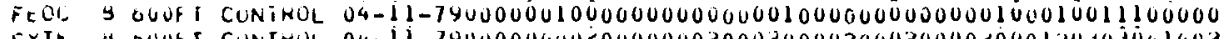

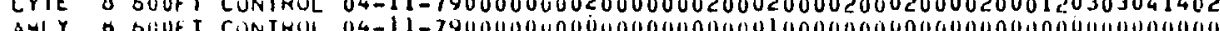

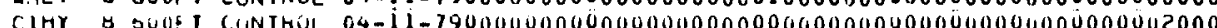
T. QATE 9 OUOF I LRUUEU 70504-11-7900000000000000000000000100000000000000000000000000 CILE $y$ OOUF I ERUUEU $7004-11-19211111<0<111110121221112111112211111110+111100110$

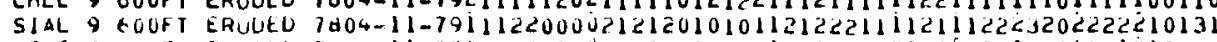
DEPI S DOUFT EROULU 7804-11-1900000010000000000000101100000000000006010001010000 AMLY 9 OUUF I EROUEU 7OUS-1!-7900000000000000000000000000000000000000010000000000 
PERCENT COVER BY SPECIES

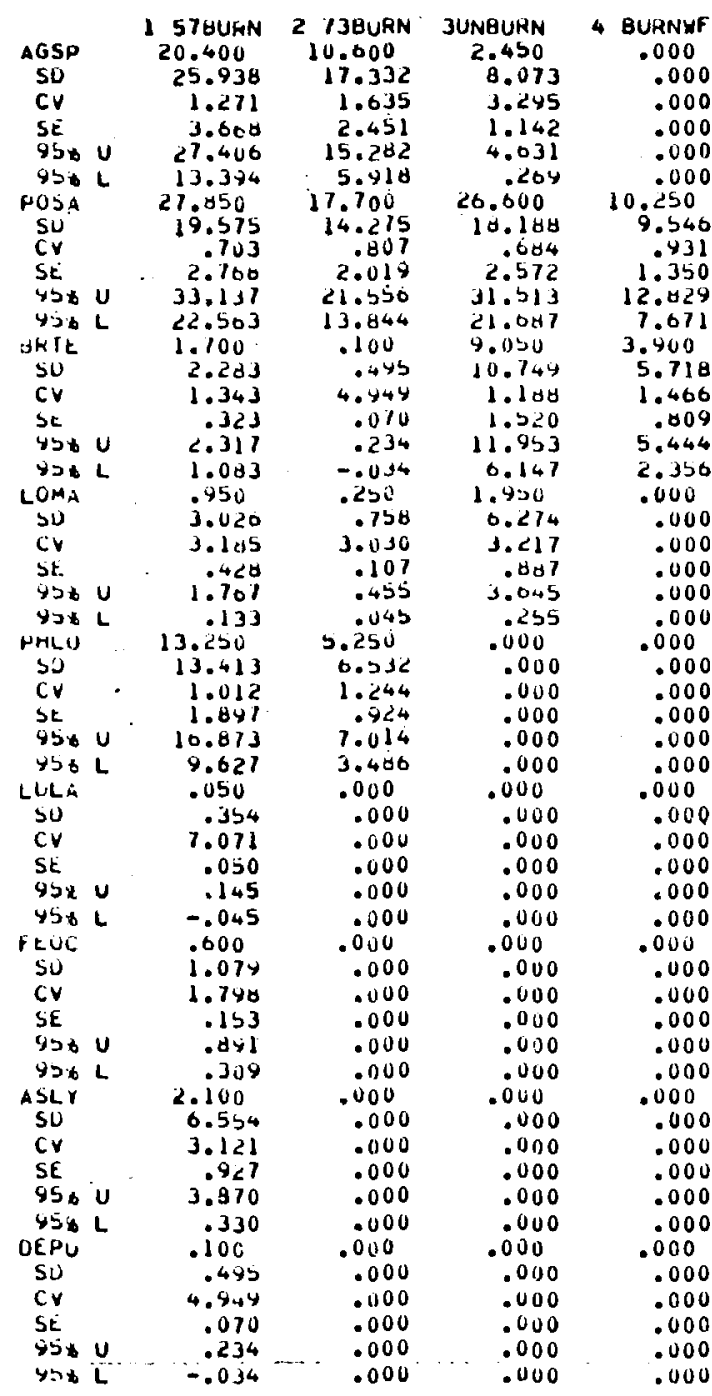

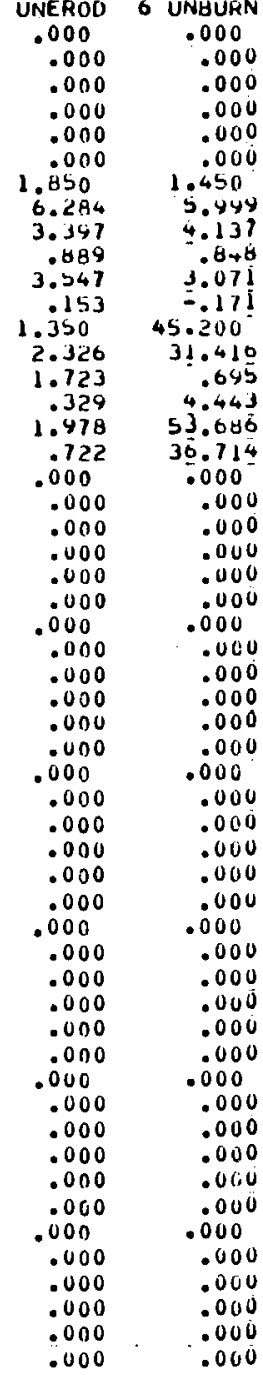

$\begin{array}{ccc}7 \text { TQGUKN } & 8 \text { CONTRL } 9 \text { EROUED } \\ .0000 & .000 & .0000\end{array}$

$\begin{array}{lllll}.000 & .000 & .000 & 3.717 & 2.387 \\ .000 & .000 & .000 & 5.705 & 3.218 \\ .000 & .000 & .000 & .684 & .389\end{array}$

MEAN STAN ERG

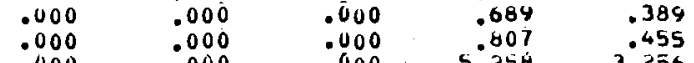

$\begin{array}{lllll}.000 & .000 & .000 & 5.258 & 3.256 \\ .000 & .000 & .000 & 2.176 & 1.518\end{array}$

.000

3.400

6.243

I. 810

3.006

.000
$000 \quad 3.006$
1.714

20.0100
19.059

$\begin{array}{ll}19.054 & 1<.612 \\ .993 & 1.693\end{array}$

$2.009 \quad 1.704$

14.036
.000

1.057
.0043
.000

$\begin{array}{ll}.000 & .000 \\ .000 & .000\end{array}$

$\begin{array}{ll}.000 & .000 \\ .000 & .000\end{array}$

.000
.000
.000
.000

.000

.000

.000

.0000

.000
.000

.000
.000

.060

.000

.000
.000
.000

.000

.350
.670

2.504

$1<4$
.567

.547
.113

.500

.000

.001
.000

.000

.0000
.000

.000
.000

.000
.000

.000

.0000 $\begin{array}{lll}6.289 & 9.600 & 2.152 \\ 3.494 & 1.777 & .504\end{array}$

$\begin{array}{rrr}.849 & 1.358 \\ 3.499 & 12.093 & .3045\end{array}$

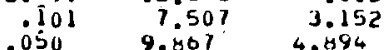

$\begin{array}{rrr}.354 & 9.867 & 4.8949 \\ 9.354 & 3.499\end{array}$

$\begin{array}{lll}.071 & 2.347 & .723 \\ .050 & 1.344 & .495\end{array}$

$.145 \quad 12.442 \quad 5.039$

$\begin{array}{lll}.0045 & 0241 & 3.444\end{array}$

$.000 \quad 1.111 \quad .725$

.000 .040 .0003

$\begin{array}{lll}.000 & .058 & .0430 \\ .0000 & 2.056 & 1.514\end{array}$

$.000 \quad 2.216 \quad 1.574$

$\begin{array}{rrr}.000 & .251 & .167 \\ .000 & .313 & .223\end{array}$

$\begin{array}{lll}.000 & 2.654 & 1.939 \\ .000 & 1.457 & 1.009\end{array}$

$.000 \quad .006$

.000 .039

$.000 \quad .006 \quad .006$

$.000 \quad-.005 \quad-.005$

$.000 \quad .106 \quad .073$

$\begin{array}{lll}.000 & .217 & .145 \\ .000 & .370 & .322\end{array}$

$.000 \quad .031 \quad .020$

$.000 \quad .164$

.000

.000

.233

.728

.03

.0430

.037
.011

.000

.000
.000
.000

.550
.008

.008
-.025
-.004

.034

. .728

.0103

.0037

.055

0.000

.020 $\begin{aligned} & 1.600 \\ & 6.249\end{aligned} 10.100-3.734$ 
PERCENT COVER BY SPECIES (Cont inued)

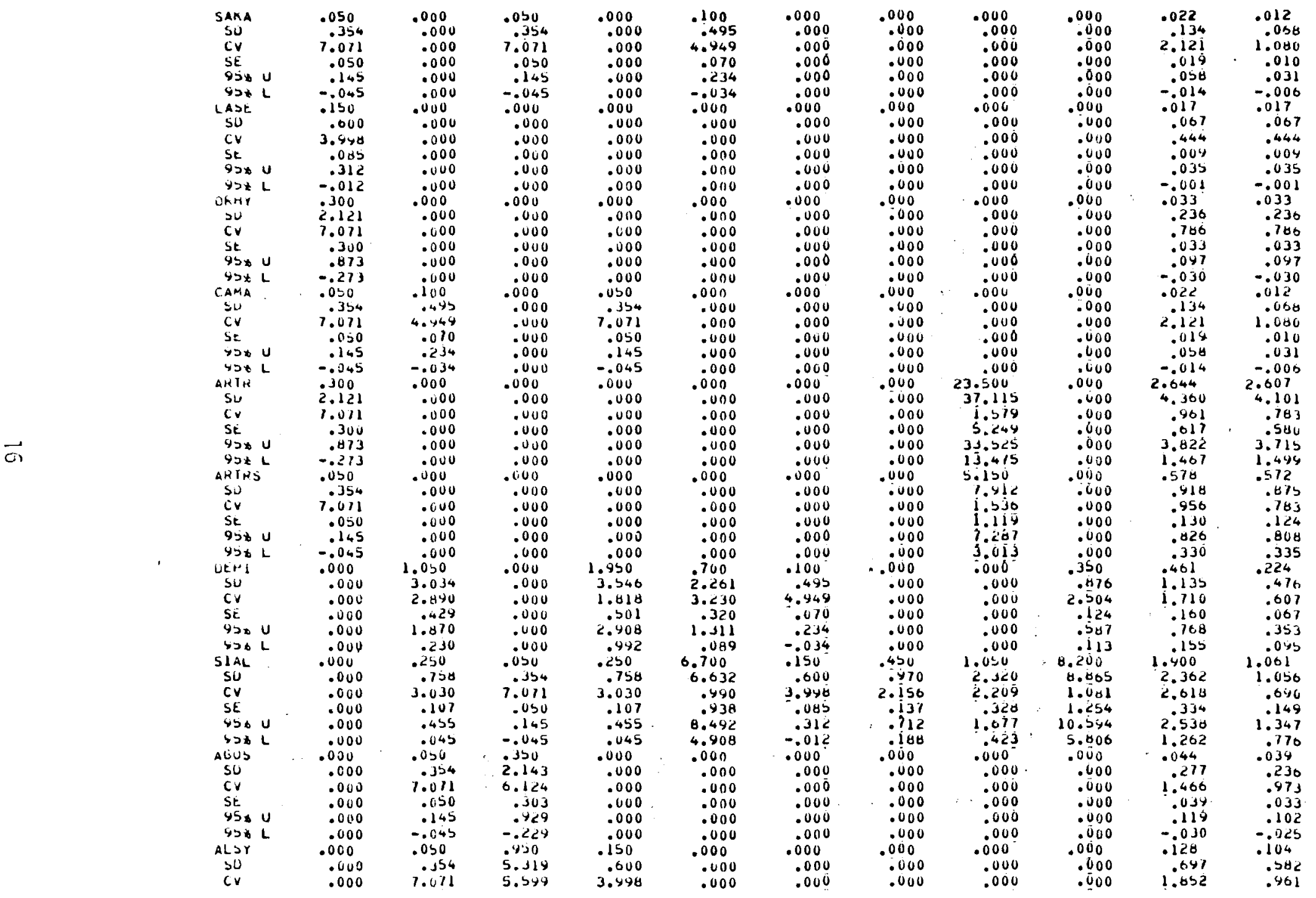


PERCENT COVER BY SPECIES (Continued)

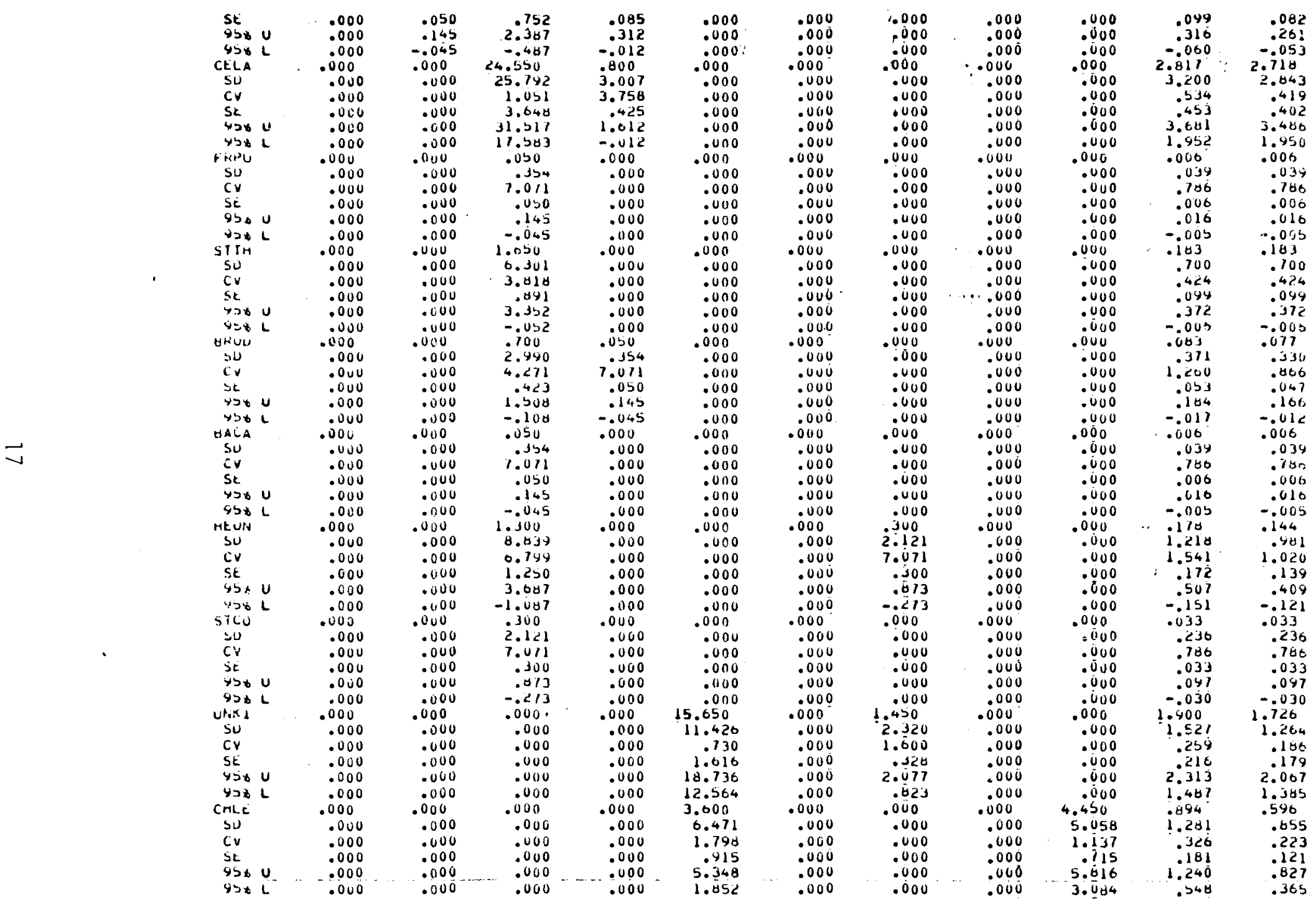


PERCENT COVER BY SPECIES (Cont inued)

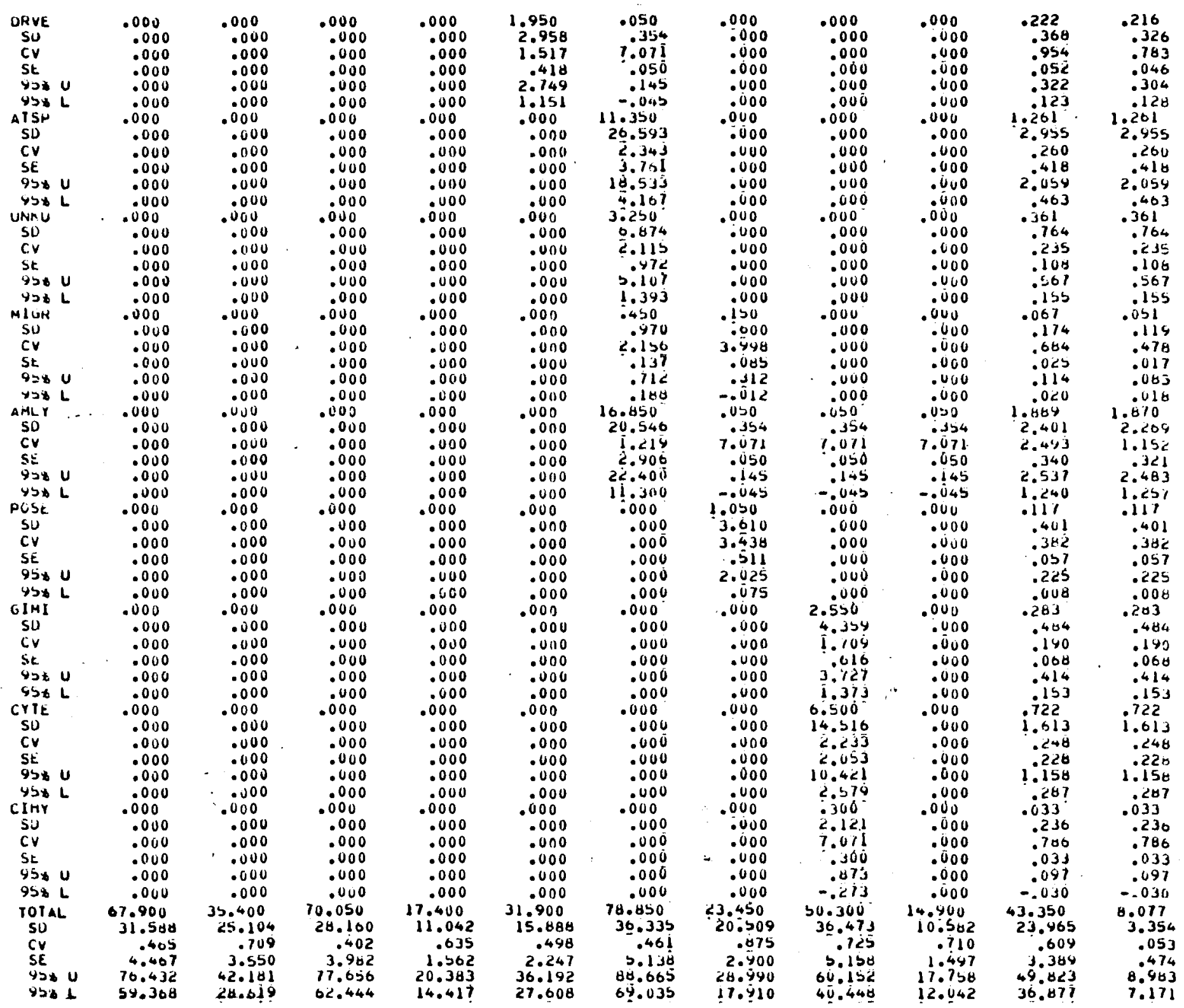




\section{SPECIES DIVERSITY}

TOTAL COVER ANU SPECIES DIVERSITY UF HILL IN ECOLOGY, 1973

\begin{tabular}{|c|c|c|c|c|c|c|c|c|c|c|c|}
\hline $\begin{array}{l}\text { TOTAL } \\
\text { N(O) } \\
\text { NOII } \\
\text { N(C) }\end{array}$ & $\begin{array}{c}15780 K N \\
01.900 \\
15.000 \\
4.210 \\
3.350\end{array}$ & $\begin{array}{c}2130 \mathrm{HKN} \\
35.400 \\
10.000 \\
3.376 \\
2.151\end{array}$ & $\begin{array}{r}3 \text { UNBURN } \\
70.050 \\
15.000 \\
4.729 \\
3.405\end{array}$ & $\begin{array}{c}\text { GURNWF } \\
17.400 \\
8.000 \\
3.220 \\
2.426\end{array}$ & $\begin{array}{c}5 \text { UHEROU } \\
31.900 \\
\text { B.000 } \\
4.459 \\
3.258\end{array}$ & $\begin{array}{c}6 \text { UNGURN } \\
7 \$ .050 \\
9.000 \\
3.278 \\
2.518\end{array}$ & $\begin{array}{r}7 \text { 7日UUKN } \\
23.450 \\
7.000 \\
1.806 \\
1.003\end{array}$ & $\begin{array}{c}\text { B CUliti.L } \\
50.200 \\
10.600 \\
5.006 \\
3.630\end{array}$ & $\begin{array}{c}9 \text { EROUEO } \\
14.400 \\
0.000 \\
2.919 \\
1.456\end{array}$ & $\begin{array}{c}\text { MEAM } \\
13.350 \\
9.778 \\
3.681 \\
7.405\end{array}$ & $\begin{array}{r}\text { SIAH ENA } \\
8.077 \\
1.077 \\
.336 \\
.0 .38\end{array}$ \\
\hline
\end{tabular}


FREQUENCY OF OCCURRENCE

\begin{tabular}{|c|c|c|c|c|c|c|}
\hline $\begin{array}{l}\triangle G S P \\
\text { POSA }\end{array}$ & $\begin{array}{c}1 \text { S7GUkN } \\
62.060 \\
90.000\end{array}$ & $\begin{array}{c}2738 \text { URN } \\
54.000 \\
90.000\end{array}$ & $\begin{array}{r}\text { 3UNEURN } \\
12.000 \\
94.0000\end{array}$ & $\begin{array}{c}4 \text { BURNWF } \\
.000 \\
86.000\end{array}$ & $\begin{array}{c}S \text { UNEROD } \\
.0000 \\
16.000\end{array}$ & $\begin{array}{r}6 \text { UNGURN } \\
10.000 \\
10.000\end{array}$ \\
\hline UKTE & 58.000 & 4.000 & 40.000 & 56.000 & 44.000 & 98.000 \\
\hline LONA & le.000 & 10.000 & $<0.000$ & .000 & .000 & .000 \\
\hline PHLO & 04.000 & 60.000 & .000 & .000 & .000 & .000 \\
\hline CWiLA & 2.000 & .000 & .000 & .000 & .000 & .000 \\
\hline ituc & 24.0100 & .000 & .000 & .000 & .000 & .000 \\
\hline Astr $r$ & 10.000 & .000 & .000 & .000 & .000 & .000 \\
\hline UEPU & 4.000 & .000 & .000 & .000 & .000 & .000 \\
\hline SAKA & 2.000 & .000 & 2.000 & .000 & 4.000 & .000 \\
\hline$L A>E$ & 0.000 & .000 & .000 & .000 & .000 & .000 \\
\hline Ekrry & C.000 & .000 & .000 & .000 & .000 & .000 \\
\hline $\operatorname{Can} A$ & 2.000 & 4.000 & .000 & 2.000 & .000 & .000 \\
\hline AKTK & 20000 & .000 & .000 & .000 & .000 & .000 \\
\hline ENIKS & 2.000 & .000 & .000 & .000 & .000 & .ute \\
\hline DERl & .000 & 22.000 & .000 & 40.000 & 18.000 & 4.000 \\
\hline SIAL & .000 & 10.000 & 2.000 & 10.000 & 78.000 & 6.400 \\
\hline AUUS & . Uuv & 2.000 & 4.000 & .060 & .000 & .000 \\
\hline$A L S Y$ & .000 & 2.000 & 10.000 & 0.000 & .000 & .000 \\
\hline CELA & .000 & .000 & 72.000 & 12.000 & .000 & .000 \\
\hline Flipu & .000 & .000 & 2.000 & .000 & .000 & .000 \\
\hline $\sin$ & .000 & .000 & 0.000 & .000 & .000 & .000 \\
\hline BNoU & .000 & .000 & 9.000 & 2.000 & .000 & .000 \\
\hline$B A C A$ & .000 & .000 & 2.000 & .000 & .000 & .000 \\
\hline HEUN & .000 & .000 & 4.000 & .000 & .000 & .000 \\
\hline$\leqslant 100$ & .000 & .000 & 2.000 & .000 & .000 & .000 \\
\hline UNM! & .000 & .000 & .000 & .000 & 98.000 & .000 \\
\hline CHLE & .000 & .000 & .000 & .000 & 66.000 & .0000 \\
\hline JWVE & .000 & .000 & .000 & .000 & 50.000 & 2.000 \\
\hline$\therefore \mathrm{TSP}$ & .000 & .000 & .000 & .000 & .000 & 26.000 \\
\hline Cimnu & .000 & .000 & .000 & .000 & .000 & 42.000 \\
\hline mlun & .000 & .000 & .000 & .000 & .000 & 18.000 \\
\hline$\therefore M L Y$ & .000 & .000 & .000 & .000 & .000 & 72.000 \\
\hline post & .000 & .000 & .000 & .000 & .000 & .000 \\
\hline GIMI & .000 & .000 & .000 & .000 & .000 & .000 \\
\hline CYIE & .000 & .000 & .000 & $.0 \cup 0$ & .000 & .000 \\
\hline Ciny & . 0 00 & .000 & .000 & .000 & .000 & .000 \\
\hline
\end{tabular}

\begin{tabular}{|c|c|c|c|c|}
\hline N & \& CONTHL & 9 EROUED & MEAN & STAN ERR \\
\hline .000 & $\therefore .000$ & .000 & 14.222 & 8.403 \\
\hline $\begin{array}{l}.000 \\
.0000\end{array}$ & 26.000 & & & 14.322 \\
\hline .000 & $\begin{array}{l}92.000 \\
.000\end{array}$ & 2.000 & $\begin{array}{r}54.778 \\
5.333\end{array}$ & $\begin{array}{r}12.541 \\
2.809\end{array}$ \\
\hline .000 & .000 & .000 & 16.000 & 10.770 \\
\hline .000 & .000 & .000 & .222 & .222 \\
\hline .000 & 14.000 & $.0 \overline{0} 0$ & 4.222 & 2.914 \\
\hline .000 & .000 & .000 & 1.778 & 1.778 \\
\hline .000 & .000 & $=000$ & $\because 444$ & .444 \\
\hline .000 & .000 & .000 & . 804 & .484 \\
\hline .000 & .000 & .000 & .607 & .667 \\
\hline .0000 & .000 & .000 & .222 & $.22 ?$ \\
\hline .000 & $.00 u$ & .000 & .689 & .464 \\
\hline .000 & 38.000 & .000 & 4.644 & 4.000 \\
\hline .000 & 40.000 & .000 & 10.222 & 9.475 \\
\hline .000 & .000 & 14.000 & 11.776 & 5.369 \\
\hline .000 & 32.000 & $8<.000$ & 26.444 & 10.008 \\
\hline .000 & .000 & .000 & .067 & .471 \\
\hline .000 & .000 & .000 & 2.000 & 1.202 \\
\hline .000 & .000 & .000 & 9.333 & 7.444 \\
\hline .000 & .000 & .060 & .222 & .222 \\
\hline .000 & .000 & .000 & . 804 & .809 \\
\hline .000 & .000 & .060 & 1.111 & .404 \\
\hline .000 & .000 & .000 & .222 & .222 \\
\hline 2.000 & .000 & .000 & & .471 \\
\hline .000 & .000 & .000 & .222 & $.2<2$ \\
\hline 8.000 & .000 & .000 & 16.222 & 11.511 \\
\hline .000 & .000 & 80.060 & 17.111 & 11.465 \\
\hline .000 & .000 & .000 & $0.0,07$ & 6.420 \\
\hline .000 & .000 & .000 & 2.084 & 2.889 \\
\hline .000 & .000 & .000 & 4.667 & 4.607 \\
\hline 000 & .000 & .000 & 2.667 & $2.0<t$ \\
\hline 2.000 & 2.000 & 2.000 & 8.607 & 7.963 \\
\hline 12.000 & .000 & .000 & 1.333 & 1.333 \\
\hline .000 & 52.000 & .000 & 5.776 & 5.778 \\
\hline . U UO & 28.000 & .000 & 3.111 & 3.111 \\
\hline .000 & 2.000 & .000 & $.2<2$ & $.2<2$ \\
\hline
\end{tabular}


PNL -3068

UC- 1$]$

$\underline{\text { DISTRIBUTION }}$

No. of

Copies

A. A. Churm

DOE Chicago Patent Attorney

9800 South Cass Avenue

Argonne, IL 60439

ONSITE

Rockwe 11 Hanford Operations

R. M. Mitche 11

46 Pacific Northwest Laboratory

27 DOE Technical Information Center

R. C. Clusen

Assistant Secretary for

Environment

DOE Office of Health and

Environmental Research

Washington, DC 20545

P. Dunaway

U.S. DOE Nevada Operations Office

P.0. Box 14100

Las Vegas, NV 89114

DOE Richland Operations Office

W. J. Bair

4. B. W. Compton

L. Kupinski

D. E. Olesen

S. L. Owzarski

D. H. McKenzie

W. H. Rickard

R. H. Sauer (25)

R. G. Schreckhise

N. M. Sherer

W. L. Temp leton

J. M. Thomas

B. E. Vaughan

5 Technical Information

2 Publishing Coordination La

H. E. Ransom 